\title{
Complemented Subspaces of Spaces Obtained by Interpolation
}

\author{
D.J.H. Garling \\ St. John's College, Cambridge CB2 1TP, England. \\ S.J. Montgomery-Smith \\ Department of Mathematics, University of Missouri, \\ Columbia, MO 65211, U.S.A.
}

\begin{abstract}
If $Z$ is a quotient of a subspace of a separable Banach space $X$, and $V$
is any separable Banach space, then there is a Banach couple $\left(A_{0}, A_{1}\right)$ such that $A_{0}$ and $A_{1}$ are isometric to $X \oplus V$, and any intermediate space obtained using the real or complex interpolation method contains a complemented subspace isomorphic to $Z$. Thus many properties of Banach spaces, including having non-trivial cotype, having the Radon-Nikodym property, and having the analytic unconditional martingale difference sequence property, do not pass to intermediate spaces.
\end{abstract}

There are many Banach space properties that pass to spaces obtained by the complex method of interpolation. For example, it is known that if a couple $\left(A_{0}, A_{1}\right)$ is such that $A_{0}$ and $A_{1}$ both have the UMD (unconditional martingale difference sequence) property, and if $A_{\theta}$ is the space obtained using the complex interpolation method with parameter $\theta$, then $A_{\theta}$ has the UMD property whenever $0<\theta<1$. Another example is type of Banach spaces: if $A_{0}$ has type $p_{0}$ and $A_{1}$ has type $p_{1}$, then $A_{\theta}$ has type $p_{\theta}$, where $1 / p_{\theta}=(1-\theta) / p_{0}+\theta / p_{1}$.

Similar results are true for the real method of interpolation. If we denote by $A_{\theta, p}$ the space obtained using the real interpolation method from a couple $\left(A_{0}, A_{1}\right)$ with parameters $\theta$ and $p$, then $A_{\theta, p}$ has the UMD property whenever $A_{0}$ and $A_{1}$ have the UMD property, $0<\theta<1$, and $1<p<\infty$. Similarly, if $A_{0}$ has type $p_{0}$ and $A_{1}$ has type $p_{1}$, then $A_{\theta, p}$ has type $p_{\theta}$, where $1 / p_{\theta}=(1-\theta) / p_{0}+\theta / p_{1}$ and $p=p_{\theta}$ (see [5] 2.g.22).

However, there are other properties for which it has been hitherto unknown whether they pass to the intermediate spaces. Examples include the Radon-Nikodym property, the AUMD (analytic unconditional martingale difference sequence) property, and having non-trivial cotype.

The second named author was supported in part by N.S.F. Grant DMS 9001796.

A.M.S. (1980) subject classification: $46 B 99$. 
This paper deals with these properties, showing that they do not pass to the intermediate spaces. Indeed, we show the surprising fact that there is a couple $\left(A_{0}, A_{1}\right)$ such that $A_{0}$ and $A_{1}$ are both isometric to $l_{1}$, but all the real or complex intermediate spaces contain a complemented subspace isomorphic to $c_{0}$. This improves a result due to Pisier, who gave an example of a couple $\left(A_{0}, A_{1}\right)$ for which $A_{0}$ is isometric to $L_{1}, A_{1}$ is isometric to a dense subspace of $c_{0}$, and $c_{0}$ is finitely represented in every intermediate space $A_{\theta}$ obtained by the complex interpolation method (see $[\mathbf{3}]$ ).

\section{Notation}

Here we outline the notation we will use about interpolation couples. The reader is referred to $[\mathbf{1}]$ or $[\mathbf{2}]$ for details.

A Banach couple is a pair of Banach spaces $\left(A_{0}, A_{1}\right)$ such that $A_{0}$ and $A_{1}$ both embed into a common topological vector space, $\Omega$, which we shall call the ambient space. Given such a couple, we define Banach spaces $A_{0}+A_{1}$ (with norm $\|x\|=\inf \left\{\left\|x_{0}\right\|_{A_{0}}+\left\|x_{1}\right\|_{A_{1}}\right.$ : $\left.x_{0} \in A_{0}, x_{1} \in A_{1}, x_{0}+x_{1}=x\right\}$ ) and $A_{0} \cap A_{1}$ (with norm $\|x\|=\max \left\{\|x\|_{A_{0}},\|x\|_{A_{1}}\right\}$ ). A map between two couples $T:\left(A_{0}, A_{1}\right) \rightarrow\left(B_{0}, B_{1}\right)$ is a linear map $T: A_{0}+A_{1} \rightarrow B_{0}+B_{1}$ such that $T\left(A_{0}\right) \subseteq B_{0}$ and $T\left(A_{1}\right) \subseteq B_{1}$, and such that $\|T\|_{A_{0} \rightarrow B_{0}},\|T\|_{A_{1} \rightarrow B_{1}}<\infty$.

An interpolation method, $I$, is a functor that takes a Banach couple $\left(A_{0}, A_{1}\right)$ to a single Banach space $A_{I}$, such that $A_{0} \cap A_{1} \subseteq A_{I} \subseteq A_{0}+A_{1}$ with

$$
c^{-1}\|x\|_{A_{0}+A_{1}} \leq\|x\|_{A_{I}} \leq c\|x\|_{A_{0} \cap A_{1}},
$$

and so that if $T:\left(A_{0}, A_{1}\right) \rightarrow\left(B_{0}, B_{1}\right)$ is a map between couples, then $T\left(A_{I}\right) \subseteq B_{I}$ with $\|T\|_{A_{I} \rightarrow B_{I}}<\infty$.

An interpolation method is called exponential with exponent $\theta$ if $0<\theta<1$, and whenever $T:\left(A_{0}, A_{1}\right) \rightarrow\left(B_{0}, B_{1}\right)$ is a map between couples, then

$$
\|T\|_{A_{I} \rightarrow B_{I}} \leq c\|T\|_{A_{0} \rightarrow B_{0}}^{1-\theta}\|T\|_{A_{1} \rightarrow B_{1}}^{\theta} .
$$

An interpolation method is called exact exponential if it is exponential and $c=1$ in inequalities (1) and (2).

The most well known interpolation methods are the real interpolation method, and the complex interpolation method. They are both exponential, and the complex interpolation method is exact exponential. Another interpolation method, parameterized by $0<\theta<1$, is the following: if $x \in A_{0} \cap A_{1}$, then let

$$
\||x|\|=\inf \left\{\sum_{i=1}^{n}\left\|x_{i}\right\|_{A_{0}}^{1-\theta}\left\|x_{i}\right\|_{A_{1}}^{\theta}: \sum_{i=1}^{n} x_{i}=x\right\} .
$$


Let $A_{\min , \theta}$ be the completion of $\left(A_{0} \cap A_{1},\||\cdot|\|\right)$. This interpolation method is exact exponential of exponent $\theta$. Furthermore, if $I$ is another exponential method of exponent $\theta$, then $\|x\|_{A_{I}} \leq c\|x\|_{A_{\min , \theta}}$, with $c=1$ if $I$ is exact exponential. (In fact, this interpolation method gives a space equivalent to the space $A_{\theta, 1}$ obtained by the real interpolation method.)

We denote by $X \oplus V$ the Banach space of ordered pairs $(x, v)$ with norm $\|(x, v)\|=$ $\|x\|+\|v\|$.

All the results given work for Banach spaces over the real or complex scalars. (Obviously the complex interpolation method will require the complex scalars.)

\section{The Main Result}

Here we present the main result of this paper.

Theorem 1. Suppose that $Z$ is a Banach space and that $Q_{i}$ is a quotient mapping onto $Z$ from a closed linear subspace $Y_{i}$ of a separable Banach space $X_{i}$, for $i=0,1$, such that the following dimensional constraints hold:

a) the dimensions of $Y_{0}$ and $Y_{1}$ are equal;

b) the codimensions of $Y_{0}$ in $X_{0}$ and $Y_{1}$ in $X_{1}$ are equal;

c) the dimensions of the kernels of $Q_{0}$ and $Q_{1}$ are equal.

Suppose further that $0<\epsilon<1$, that $0<\theta_{0} \leq \theta_{1}<1$ and that $V_{0}$ and $V_{1}$ are any infinite dimensional separable Banach spaces.

Then there is a Banach couple $\left(A_{0}, A_{1}\right)$, a subspace $W$ of $A_{0}+A_{1}$, and linear maps $P: W \rightarrow Z$ and $E: Z \rightarrow W$ with the following properties:

i) $A_{0}$ is isometric to $X_{0} \oplus V_{0}$, and $A_{1}$ is isometric to $X_{1} \oplus V_{1}$;

ii) $P \circ E=\operatorname{Id}_{Z}$;

if $I$ is an exponential interpolation method of exponent $\theta$, then

iii) $A_{I} \subseteq W$ and $\|P\|_{A_{I} \rightarrow Z}<\infty$ (with $\|P\|_{A_{I} \rightarrow Z}<1+\epsilon / 3$ if $I$ is exact and $\theta_{0} \leq \theta \leq \theta_{1}$ );

iv) $E(Z) \subseteq A_{I}$ and $\|E\|_{Z \rightarrow A_{I}}<\infty$ (with $\|E\|_{Z \rightarrow A_{I}}<1+\epsilon / 3$ if $I$ is exact and $\theta_{0} \leq \theta \leq$ $\left.\theta_{1}\right)$.

Thus $Z$ is isomorphic to a complemented subspace of $A_{I}$. (If $I$ is exact and $\theta_{0} \leq \theta \leq \theta_{1}$, then $Z$ is $(1+\epsilon)$ isomorphic to a $(1+\epsilon)$-complemented subspace of $A_{I}$.)

This has the following corollaries.

Corollary. Given any separable Banach space $X$, there is a Banach couple $\left(A_{0}, A_{1}\right)$ such that $A_{0}$ and $A_{1}$ are isometric to $l_{1}$, and for every exponential interpolation method $I$, the intermediate space $A_{I}$ contains a complemented subspace isomorphic to $X$. 
Corollary. Given any separable Banach space $X$, there is a Banach couple $\left(A_{0}, A_{1}\right)$ such that $A_{0}$ and $A_{1}$ are isometric to $C([0,1])$, and for every exponential interpolation method $I$, the intermediate space $A_{I}$ contains a complemented subspace isomorphic to $X$.

Corollary. The following properties do not pass to intermediate spaces by any exponential interpolation methods, in the sense that there is a couple $\left(A_{0}, A_{1}\right)$ such that $A_{0}$ and $A_{1}$ both have the property, but none of the intermediate spaces do:

i) the Radon-Nikodym property;

ii) the analytic Radon-Nikodym property;

iii) having non-trivial cotype;

iv) the AUMD property;

v) having a dual with non-trivial cotype;

vi) having a dual with the AUMD property.

Note that parts (v) and (vi) of the above corollary follow because $C([0,1])^{*}$ is finitely represented in $l_{1}$.

\section{A More Elementary Result}

Before presenting the proof of Theorem 1, we first prove a more elementary result that is, in fact, a corollary of Theorem 1. The reason for this is that the proof of Theorem 1 involves many technicalities, disguising the essential idea of the proof, which is very simple.

Theorem 2. There is a Banach couple $\left(A_{0}, A_{1}\right)$ such that $A_{0}$ and $A_{1}$ are isometric to $l_{1}$, and for every exponential interpolation method $I$, the intermediate space $A_{I}$ contains a complemented subspace isomorphic to $c_{0}$.

Proof: Let $e_{n}$ be the unit vectors in $l_{1}$ and $c_{0}$, and let $r_{n}$ be an enumeration of the "corners of the unit cube' in $c_{0}$, that is, all vectors of the form $( \pm 1, \pm 1, \ldots, \pm 1,0,0,0, \ldots)$. Let $\epsilon_{n}$ be the sequence of numbers defined by

$$
\epsilon_{n}=\left(2+\left\|r_{n}\right\|_{1}\right)^{-n} .
$$

(Any sequence of numbers tending sufficiently rapidly to zero will do.) We form an ambient space $\Omega=c_{0} \oplus l_{1} \oplus l_{1}$. We define subspaces

$$
\begin{aligned}
& A_{0}=\left\{\left(\sum_{n} a_{n} e_{n}+b_{n} r_{n}, \sum_{n} \epsilon_{n} b_{n} e_{n}, \sum_{n} c_{n} e_{n}\right):\left(a_{n}\right),\left(b_{n}\right),\left(c_{n}\right) \in l_{1}\right\} \\
& A_{1}=\left\{\left(\sum_{n} a_{n} e_{n}+c_{n} r_{n}, \sum_{n} b_{n} e_{n}, \sum_{n} \epsilon_{n} c_{n} e_{n}\right):\left(a_{n}\right),\left(b_{n}\right),\left(c_{n}\right) \in l_{1}\right\},
\end{aligned}
$$


with norms

$$
\begin{aligned}
& \left\|\left(\sum_{n} a_{n} e_{n}+b_{n} r_{n}, \sum_{n} \epsilon_{n} b_{n} e_{n}, \sum_{n} c_{n} e_{n}\right)\right\|_{A_{0}}=\sum_{n}\left(\left|a_{n}\right|+\left|b_{n}\right|+\left|c_{n}\right|\right) \\
& \left\|\left(\sum_{n} a_{n} e_{n}+c_{n} r_{n}, \sum_{n} b_{n} e_{n}, \sum_{n} \epsilon_{n} c_{n} e_{n}\right)\right\|_{A_{1}}=\sum_{n}\left(\left|a_{n}\right|+\left|b_{n}\right|+\left|c_{n}\right|\right) .
\end{aligned}
$$

The idea is that the unit balls of $A_{0}$ and $A_{1}$ are 'slightly perturbed' versions of the unit balls of $c_{0}$, where the perturbations for $A_{0}$ and $A_{1}$ go in different directions. These perturbations cause the unit balls of $A_{0}$ and $A_{1}$ to be convex hulls of linearly independent vectors, and hence they are affine to the unit ball of $l_{1}$. The size of these perturbations is controlled by the quantities $\epsilon_{n}$. The vectors that are used to perturb the unit ball of $A_{0}$ are also contained in $A_{1}$ with no control on their size. Similarly, the perturbing vectors of $A_{1}$ are contained in $A_{0}$. Thus, when we form the intermediate space $A_{I}$, the perturbations in $A_{0}$ are 'swamped out' or 'washed away' by the vectors in $A_{1}$, and similarly for the perturbations in $A_{1}$, and we are left with a complemented copy of $c_{0}$. So much for the idea-now we give the proof.

We define a projection $P: \Omega \rightarrow c_{0}$ by

$$
P(x, y, z)=x
$$

It is easy to see that $\|P\|_{A_{i} \rightarrow c_{0}} \leq 1$ for $i=0,1$, and hence $\|P\|_{A_{I} \rightarrow c_{0}}<\infty$ for any interpolation method $I$.

We define an embedding $E: c_{0} \rightarrow \Omega$ by

$$
E(x)=(x, 0,0)
$$

We prove that for every exponential interpolation method that $E(x) \in A_{I}\left(x \in c_{0}\right)$, with $\|E\|_{c_{0} \rightarrow A_{I}}<\infty$. To do this, it is sufficient to show that there is a constant $c$, depending on the exponential interpolation method $I$ only, such that for every $n \geq 1$ we have

$$
\left\|\left(r_{n}, 0,0\right)\right\|_{A_{I}} \leq c
$$

We first note the following facts.

$$
\begin{gathered}
\left\|\left(r_{n}, \epsilon_{n} e_{n}, 0\right)\right\|_{A_{0}}=\left\|\left(r_{n}, 0, \epsilon_{n} e_{n}\right)\right\|_{A_{1}}=1 \\
\left\|\left(0,0, \epsilon_{n} e_{n}\right)\right\|_{A_{0}}=\left\|\left(0, \epsilon_{n} e_{n}, 0\right)\right\|_{A_{1}}=\epsilon_{n}
\end{gathered}
$$




$$
\begin{aligned}
& \text { SPACES OBTAINED By INTERPolation } \\
& \left\|\left(r_{n}, 0,0\right)\right\|_{A_{0}}=\left\|\left(r_{n}, 0,0\right)\right\|_{A_{1}}=\left\|r_{n}\right\|_{1} .
\end{aligned}
$$

Let us suppose that $I$ is of exponent $\theta$. We shall give the details in the case where $I$ is exact exponential: a similar argument works in the general case, with less control of the constants. We have the following inequalities.

$$
\left\|\left(r_{n}, 0,0\right)\right\|_{A_{I}} \leq\left\|\left(r_{n}, \epsilon_{n} e_{n}, \epsilon_{n} e_{n}\right)\right\|_{A_{I}}+\left\|\left(0, \epsilon_{n} e_{n}, 0\right)\right\|_{A_{I}}+\left\|\left(0,0, \epsilon_{n} e_{n}\right)\right\|_{A_{I}},
$$

and

$$
\begin{aligned}
\left\|\left(r_{n}, \epsilon_{n} e_{n}, \epsilon_{n} e_{n}\right)\right\|_{A_{I}} \leq & \left\|\left(r_{n}, \epsilon_{n} e_{n}, \epsilon_{n} e_{n}\right)\right\|_{A_{0}}^{1-\theta}\left\|\left(r_{n}, \epsilon_{n} e_{n}, \epsilon_{n} e_{n}\right)\right\|_{A_{1}}^{\theta} \\
\leq & \left(\left\|\left(r_{n}, \epsilon_{n} e_{n}, 0\right)\right\|_{A_{0}}+\left\|\left(0,0, \epsilon_{n} e_{n}\right)\right\|_{A_{0}}\right)^{1-\theta} \\
& \times\left(\left\|\left(r_{n}, 0, \epsilon_{n} e_{n}\right)\right\|_{A_{1}}+\left\|\left(0, \epsilon e_{n}, 0\right)\right\|_{A_{1}}\right)^{\theta} \\
& \leq\left(1+\epsilon_{n}\right)^{1-\theta}\left(1+\epsilon_{n}\right)^{\theta} \\
& \leq\left(1+\epsilon_{n}\right),
\end{aligned}
$$

and

$$
\left\|\left(0, \epsilon_{n} e_{n}, 0\right)\right\|_{A_{I}} \leq\left\|\left(0, \epsilon_{n} e_{n}, 0\right)\right\|_{A_{0}}^{1-\theta}\left\|\left(0, \epsilon_{n} e_{n}, 0\right)\right\|_{A_{1}}^{\theta} .
$$

But

$$
\begin{aligned}
\left\|\left(0, \epsilon_{n} e_{n}, 0\right)\right\|_{A_{0}} & \leq\left\|\left(r_{n}, \epsilon_{n} e_{n}, 0\right)\right\|_{A_{0}}+\left\|\left(r_{n}, 0,0\right)\right\|_{A_{0}} \\
& \leq 1+\left\|r_{n}\right\|_{1},
\end{aligned}
$$

and so

$$
\left\|\left(0, \epsilon_{n} e_{n}, 0\right)\right\|_{A_{I}} \leq\left(1+\left\|r_{n}\right\|_{1}\right)^{1-\theta} \epsilon_{n}^{\theta} .
$$

Similarly

$$
\left\|\left(0,0, \epsilon_{n} e_{n}\right)\right\|_{A_{I}} \leq\left(1+\left\|r_{n}\right\|_{1}\right)^{\theta} \epsilon_{n}^{1-\theta} .
$$

Therefore

$$
\left\|\left(r_{n}, 0,0\right)\right\|_{A_{I}} \leq 1+\epsilon_{n}+\left(1+\left\|r_{n}\right\|_{1}\right)^{1-\theta} \epsilon_{n}^{\theta}+\left(1+\left\|r_{n}\right\|_{1}\right)^{\theta} \epsilon_{n}^{1-\theta} .
$$

By our choice of $\epsilon_{n}$, this is bounded by some constant $c$ that depends only upon $\theta$.

Thus we have bounded maps

$$
P: A_{I} \rightarrow c_{0} \quad E: c_{0} \rightarrow A_{I}
$$

such that $P \circ I=\mathrm{Id}_{c_{0}}$, and hence $c_{0}$ is isomorphic to a complemented subspace of $A_{I}$.

Finally, it is very clear that $A_{0}$ and $A_{1}$ are both isometric to $l_{1}$. 


\section{The Proof of Theorem 1}

In the sequel, we will make much use of biorthogonal systems. A biorthogonal system of a Banach space $X$ is a sequence $\left(x_{n} ; \xi_{n}\right) \in X \times X^{*}$ such that $\xi_{n}\left(x_{m}\right)=0$ if $n \neq m$ and 1 if $n=m$. A biorthogonal system is called fundamental if $\overline{\operatorname{span}}\left\{x_{n}\right\}=X$, and it is called total if $x=0$ whenever $\xi_{n}(x)=0$ for all $n$. (Of course, if $X$ is finite dimensional, then the sequences will be finite.) A result of Markushevich shows that every separable Banach space has a total, fundamental biorthogonal system (see [6] or [4] 1.f.3).

We shall need the following proposition and its corollary.

Lemma 3. Let $Z$ be a quotient of a separable Banach space $Y$ by the quotient map $Q$ with kernel $K$. Suppose that $\left(k_{p} ; \kappa_{p}^{\prime}\right)$ is a total fundamental biorthogonal system for $K$, and that $\left(z_{n} ; \zeta_{n}\right)$ is a total fundamental biorthogonal system for $Z$. Then there are sequences $\left(y_{n}\right)$ in $Y$, and $\left(\psi_{n}\right)$ and $\left(\kappa_{p}\right)$ in $Y^{*}$, for which the following hold:

i) $\left(y_{n}, k_{p} ; \psi_{n}, \kappa_{p}\right)$ is a total fundamental biorthogonal system for $Y$;

ii) $Q\left(y_{n}\right)=z_{n}$ and $\zeta_{n} \circ Q=\psi_{n}$ for all $n$;

iii) $y \in K$ if and only if $\psi_{n}(y)=0$ for all $n$.

Proof: Choose $\tilde{y}_{n} \in Y$ such that $Q\left(\tilde{y}_{n}\right)=z_{n}$, and let $\psi_{n}=\zeta_{n} \circ Q$. Using the Hahn-Banach Theorem, extend $\kappa_{p}^{\prime}$ to $\kappa_{p}$ on $Y$ so that $\kappa_{p}\left(\tilde{y}_{n}\right)=0$ for $n<p$. Set

$$
y_{n}=\tilde{y}_{n}-\sum_{m=1}^{n} \kappa_{m}\left(\tilde{y}_{n}\right) k_{m} .
$$

It is now straightforward to verify the conclusions of the lemma.

Lemma 4. Let $Y$ be a closed subspace of a separable Banach space $X$, and let $\left(y_{n} ; \psi_{n}^{\prime}\right)$ be a total fundamental biorthogonal system for $Y$. Then there are sequences $\left(x_{m}\right)$ in $X$, and $\left(\xi_{m}\right)$ and $\left(\psi_{n}\right)$ in $X^{*}$, such that $\left(x_{m}, y_{n} ; \xi_{m}, \psi_{n}\right)$ is a total fundamental biorthogonal system for $X$, and such that an element $x$ in $X$ belongs to $Y$ if and only if $\xi_{m}(x)=0$ for all $m$.

Proof: By Markushevich's result, $Y$ has a total fundamental system $\left(y_{n} ; \psi_{n}^{\prime}\right)$, and $X / Y$ has a total fundamental system $\left(z_{m} ; \zeta_{m}\right)$. Then the result follows by applying Lemma 3 to the quotient mapping $Q: X \rightarrow X / Y$. 
Proof of Theorem 1: We begin by defining some biorthogonal systems. First, let $\left(z_{n}, \zeta_{n}\right)$ be a total fundamental biorthogonal system for $Z$, with $\left\|\zeta_{n}\right\|=1$ for all $n$. Using Lemmas 3 and 4 , for $i=0,1$, we can find total fundamental biorthogonal systems

$$
\left(x_{m}^{i}, y_{n}^{i}, k_{p}^{i} ; \xi_{m}^{i}, \psi_{n}^{i}, \kappa_{p}^{i}\right)
$$

for $X_{i}$ with the following properties:

i) $Q_{i}\left(y_{n}^{i}\right)=z_{n}$ and $\zeta_{n} \circ Q_{i}=\psi_{n}^{i}$ for all $n$;

ii) $Y_{i}=\left\{x \in X_{i}: \xi_{m}^{i}(x)=0\right.$ for all $\left.m\right\}$;

iii) $\left(y_{n}^{i}, k_{p}^{i} ;\left.\psi_{n}^{i}\right|_{Y_{i}},\left.\kappa_{p}^{i}\right|_{Y_{i}}\right)$ is a total fundamental biorthogonal system for $Y_{i}$;

iv) $\left(k_{p}^{i} ;\left.\kappa_{p}^{i}\right|_{K_{i}}\right)$ is a total fundamental biorthogonal system for $K_{i}$, the kernel of $Q_{i}$;

v) $\left\|\xi_{m}^{i}\right\|=\left\|\psi_{n}^{i}\right\|=\left\|\kappa_{p}^{i}\right\|=1$ for all $m, n$ and $p$.

We also choose total fundamental biorthogonal systems $\left(v_{q}^{i} ; \phi_{q}^{i}\right)$ for $V_{i}$, with $\left\|\phi_{q}^{i}\right\|=1$ for all $q$. If any of these systems is finite, then we extend it to an infinite system by including zero terms.

Next we define some sequences of rapidly decreasing numbers. Let $\nu_{n}$ and $\mu_{n}$ be sequences of positive numbers for which

$$
\begin{aligned}
\sum L_{n} \nu_{n} & \leq \epsilon / 12, \\
\max \left\{\mu_{n}^{\theta} L_{n}, \mu_{n}^{1-\theta} L_{n}\right\} & \leq \nu_{n} \quad \text { for } \theta_{0} \leq \theta \leq \theta_{1},
\end{aligned}
$$

and

$$
\sum \mu_{n}^{\theta} L_{n}<\infty \quad \text { for } 0<\theta<1
$$

where

$$
L_{n}=\max \left\{\left\|x_{n}^{i}\right\|,\left\|y_{n}^{i}\right\|,\left\|k_{p}^{i}\right\|,\left\|v_{2 n-1}^{i}\right\|,\left\|v_{2 n}^{i}\right\|, 1: i=0,1\right\} .
$$

Now we define the Banach couple. We take as the ambient space $\Omega=l_{\infty} \oplus l_{\infty} \oplus l_{\infty} \oplus$ $l_{\infty} \oplus l_{\infty}$. We define linear maps $M_{i}: X_{i} \oplus V_{i} \rightarrow \Omega$ in the following way:

$$
\begin{aligned}
& M_{0}(x, v)=\left(\left(\psi_{n}^{0}(x)\right),\left(\xi_{m}^{0}(x)\right),\left(\mu_{p} \kappa_{p}^{0}(x)\right),\left(\mu_{q} \phi_{2 q-1}^{0}(v)\right),\left(\phi_{2 q}^{0}(v)\right)\right), \\
& M_{1}(x, v)=\left(\left(\psi_{n}^{1}(x)\right),\left(\mu_{q} \phi_{2 q-1}^{1}(v)\right),\left(\phi_{2 q}^{1}(v)\right),\left(\xi_{m}^{1}(x)\right),\left(\mu_{p} \kappa_{p}^{1}(x)\right),\right) .
\end{aligned}
$$

The important feature here is the interchange in the order of the terms. We note that $M_{0}$ and $M_{1}$ are one-one maps into $\Omega$. For $i=0,1$, we set $A_{i}=M_{i}\left(X_{i} \oplus V_{i}\right)$ with their norms inherited from the domains. We take $W$ to be the linear span of all the exponential interpolation spaces $A_{I}$. 
We shall now go through the details for the case when $I$ is exact and $\theta_{0} \leq \theta \leq \theta_{1}$. Otherwise the arguments are similar, with less control of the constants. Let $e_{n}$ denote the $n$th unit vector in $l_{\infty}$. Then

$$
\left(0, \mu_{n} e_{n}, 0,0,0\right)=\mu_{n} M_{0}\left(x_{n}^{0}\right)=M_{1}\left(v_{2 n-1}^{1}\right),
$$

so that

$$
\left\|\left(0, \mu_{n} e_{n}, 0,0,0\right)\right\|_{A_{0}} \leq \mu_{n} L_{n}
$$

and

$$
\left\|\left(0, \mu_{n} e_{n}, 0,0,0\right)\right\|_{A_{1}} \leq L_{n}
$$

Hence

$$
\left\|\left(0, \mu_{n} e_{n}, 0,0,0\right)\right\|_{A_{I}} \leq \mu_{n}^{1-\theta} L_{n} \leq \nu_{n}
$$

Similarly,

$$
\begin{aligned}
\left\|\left(0,0, \mu_{n} e_{n}, 0,0\right)\right\|_{A_{I}} & \leq \nu_{n}, \\
\left\|\left(0,0,0, \mu_{n} e_{n}, 0\right)\right\|_{A_{I}} & \leq \nu_{n}
\end{aligned}
$$

and

$$
\left\|\left(0,0,0,0, \mu_{n} e_{n}\right)\right\|_{A_{I}} \leq \nu_{n}
$$

We define linear functionals on $\Omega$ as follows: if $t=(h, a, b, c, d) \in \Omega$, then let $\eta_{n}(t)=$ $h_{n}, \alpha_{n}(t)=a_{n}, \beta_{n}(t)=b_{n}, \gamma_{n}(t)=c_{n}$ and $\delta_{n}(t)=d_{n}$. Then we have that

$$
\left\|\alpha_{n}\right\|_{A_{0}^{*}}=1
$$

and

$$
\left\|\alpha_{n}\right\|_{A_{1}^{*}}=\mu_{n}
$$

and so

$$
\left\|\alpha_{n}\right\|_{A_{I}^{*}} \leq \mu_{n}^{\theta} \leq \nu_{n}
$$

Similarly, $\left\|\beta_{n}\right\|_{A_{I}^{*}} \leq \nu_{n},\left\|\gamma_{n}\right\|_{A_{I}^{*}} \leq \nu_{n}$ and $\left\|\delta_{n}\right\|_{A_{I}^{*}} \leq \nu_{n}$.

Now we define $E: Z \rightarrow \Omega$ by $E(z)=\left(\left(\zeta_{n}(z)\right), 0,0,0,0\right)$. We assert that $E(z) \in A_{I}$, with $\|E(z)\|_{A_{I}} \leq(1+\epsilon / 3)\|z\|$. To show this, we can suppose that $\|z\|<1$. For $i=0,1$, choose $y_{i} \in Y_{i}$ such that $Q_{i}\left(y_{i}\right)=z$ and $\left\|y_{i}\right\| \leq 1$. Let

$$
v_{0}=\sum_{p} \mu_{p} \kappa_{p}^{1}\left(y_{1}\right) v_{2 p}^{0} \quad \text { and } \quad v_{1}=\sum_{p} \mu_{p} \kappa_{p}^{0}\left(y_{0}\right) v_{2 p}^{1}
$$


It follows from the choice of $\mu_{p}$ that these sums converge in $V_{0}$ and $V_{1}$, and that $\left\|v_{0}\right\| \leq \epsilon / 12$ and $\left\|v_{1}\right\| \leq \epsilon / 12$. Now

$$
\begin{aligned}
M_{0}\left(y_{0}, v_{0}\right) & =M_{1}\left(y_{1}, v_{1}\right) \\
& =\left(\left(\zeta_{n}(z)\right), 0,\left(\mu_{p} \kappa_{p}^{0}\left(y_{0}\right)\right), 0,\left(\mu_{p} \kappa_{p}^{1}\left(y_{1}\right)\right)\right) \\
& =w,
\end{aligned}
$$

say, so that $w \in A_{I}$ with $\|w\|_{A_{I}} \leq 1+\epsilon / 12$. But

$$
\left\|\left(0,0,\left(\mu_{p} \kappa_{p}^{0}\left(y_{0}\right)\right), 0,0\right)\right\|_{A_{I}} \leq \sum \mu_{p} L_{p} \leq \epsilon / 12
$$

and similarly,

$$
\left\|\left(0,0,0,0,\left(\mu_{p} \kappa_{p}^{0}\left(y_{0}\right)\right)\right)\right\|_{A_{I}} \leq \epsilon / 12 .
$$

Hence $E(z) \in A_{I}$ with $\|E(z)\| \leq 1+\epsilon / 3$, as desired.

Now we turn to the problem of defining $P$. To do this, we will first show how we may consider $Z$ as a subspace of what we shall call $X_{0} \oplus_{Q} X_{1}$. We let

$$
D=\left\{\left(y_{0}, y_{1}\right): y_{i} \in Y_{i}, Q_{0}\left(y_{0}\right)+Q_{1}\left(y_{1}\right)=0\right\},
$$

and let $X_{0} \oplus_{Q} X_{1}=X_{0} \oplus X_{1} / D$. We denote by $Q_{D}$ the quotient map from $X_{0} \oplus X_{1}$ to $X_{0} \oplus_{Q} X_{1}$. We define $J: Z \rightarrow X_{0} \oplus_{Q} X_{1}$ by setting $J\left(Q_{0}(y)\right)=Q_{D}(y, 0)$ for $y \in Y_{0}$. It is easy to see that $J$ is well defined, and that $J\left(Q_{1}(y)\right)=Q_{D}(0, y)$ for $y \in Y_{1}$.

Suppose that $w=Q_{D}\left(x_{0}, x_{1}\right) \in X_{0} \oplus_{Q} X_{1}$. Then it is easy to verify that $\pi_{m}^{0}(w)=$ $\xi_{m}^{0}\left(x_{0}\right)$ and $\pi_{m}^{1}(w)=\xi_{m}^{1}\left(x_{1}\right)$ are well defined maps. It is also easy to check that $Z$ is isometric to the space

$$
J(Z)=\left\{w: \pi_{m}^{0}(w)=\pi_{m}^{1}(w)=0\right\} .
$$

Now, we will define two bounded operators $S$ and $T$ from $A_{I}$ to $X_{0} \oplus_{Q} X_{1}$. First we will concentrate on $S$, which will, in fact, be defined on $A_{0}+A_{1}$.

If $t=M_{0}(x, v) \in A_{0}$, we let

$$
R_{0}(t)=\sum \mu_{m} \phi_{2 m-1}^{0}(v) x_{m}^{1}
$$

By the choice of $\mu_{m}$, the sum converges in $X_{1}$ with $\left\|R_{0}(t)\right\| \leq\|v\|$. Thus if we set $S_{0}(t)=Q_{D}\left(x, R_{0}(t)\right)$, then $S_{0}$ is a norm decreasing map from $A_{0}$ to $X_{0} \oplus_{Q} X_{1}$. We define $S_{1}$ in a similar way. 
Now, if $t=M_{0}\left(x_{0}, v_{0}\right)=M_{1}\left(x_{1}, v_{1}\right) \in A_{0} \cap A_{1}$, then

$$
\xi_{m}^{0}\left(x_{0}\right)=\mu_{m} \phi_{2 m-1}^{1}\left(v_{1}\right)=\xi_{m}^{0}\left(R_{1}(t)\right)
$$

and

$$
\xi_{m}^{1}\left(x_{1}\right)=\mu_{m} \phi_{2 m-1}^{0}\left(v_{0}\right)=\xi_{m}^{1}\left(R_{0}(t)\right),
$$

so that $x_{0}-R_{1}(t) \in Y_{0}$ and $x_{1}-R_{0}(t) \in Y_{1}$. Further,

$$
\zeta_{n}\left(Q_{0}\left(x_{0}-R_{1}(t)\right)\right)=\psi_{n}^{0}\left(x_{0}-R_{1}(t)\right)=\psi_{n}^{0}\left(x_{0}\right),
$$

and similarly

$$
\zeta_{n}\left(Q_{1}\left(x_{1}-R_{0}(t)\right)\right)=\psi_{n}^{1}\left(x_{1}\right) .
$$

Then, since $\psi_{n}^{0}\left(x_{0}\right)=\psi_{n}^{1}\left(x_{1}\right)=\eta_{n}(t)$, and since $\left(\zeta_{n}\right)$ is total, it follows that $Q_{0}\left(x_{0}-\right.$ $\left.R_{1}(t)\right)=Q_{1}\left(x_{1}-R_{0}(t)\right)$. Thus we see that

$$
S_{0}(t)=Q_{D}\left(x_{0}-R_{1}(t)\right)=Q_{D}\left(x_{1}-R_{0}(t)\right)=S_{1}(t)
$$

and so we may define $S: A_{0}+A_{1} \rightarrow X_{0} \oplus_{Q} X_{1}$ by setting $\left.S\right|_{X_{0}}=S_{0}$ and $\left.S\right|_{X_{1}}=S_{1}$. Clearly, $S$ maps $A_{I}$ into $X_{0} \oplus_{Q} X_{1}$ with $\|S\|_{A_{I} \rightarrow X_{0} \oplus_{Q} X_{1}} \leq 1$.

Now we establish the effect of $\pi_{m}^{0}$ and $\pi_{m}^{1}$ on $S$. If $t=(h, a, b, c, d)=M_{0}(x, v) \in A_{0}$, then

$$
\pi_{m}^{0}\left(S_{0}(t)\right)=\pi_{m}^{0}\left(Q_{D}\left(x-R_{0}(t)\right)\right)=\xi_{m}^{0}(x)=a_{m}
$$

and

$$
\pi_{m}^{1}\left(S_{0}(t)\right)=\pi_{m}^{0}\left(Q_{D}\left(x-R_{0}(t)\right)\right)=\xi_{m}^{0}\left(R_{0}(t)\right)=\mu_{m} \phi_{2 m-1}(v)=c_{m} .
$$

Similarly, if $t=(h, a, b, c, d)=M_{1}(x, v) \in A_{1}$, then $\pi_{m}^{0}\left(S_{1}(t)\right)=a_{m}$ and $\pi_{m}^{1}\left(S_{1}(t)\right)=c_{m}$. Thus, if $t=(h, a, b, c, d) \in A_{I}$, then

$$
\pi_{m}^{0}(S(t))=a_{m} \quad \text { and } \quad \pi_{m}^{1}(S(t))=c_{m}
$$

Next, we define $T: A_{I} \rightarrow X_{0} \oplus_{Q} X_{1}$ by

$$
T(t)=Q_{D}\left(\sum \alpha_{m}(t) x_{m}^{0}, \sum \gamma_{m}(t) x_{m}^{1}\right)
$$

Note that the sums converge, and that

$$
\|T\| \leq \sum \nu_{m}\left(\left\|x_{m}^{0}\right\|+\left\|x_{m}^{1}\right\|\right) \leq \epsilon / 6
$$


We see that, if $t=(h, a, b, c, d)$, then $\pi_{m}^{0}(T(t))=a_{m}$ and $\pi_{m}^{1}(T(t))=c_{m}$.

Hence $(S-T)(t) \in J(Z)$. So now we set $P=J^{-1} \circ(S-T)$. We have that $\|P\|_{A_{I} \rightarrow Z} \leq$ $1+\epsilon / 3$, and

$$
\begin{aligned}
P \circ E\left(z_{n}\right) & =P\left(u_{n}, 0,0,0,0\right) \\
& =J^{-1} \circ(S-T)\left(u_{n}, 0,0,0,0\right) \\
& =J^{-1} \circ S\left(u_{n}, 0,0,0,0\right) \\
& =J^{-1} \circ S_{0} \circ M_{0}\left(y_{n}^{0}, 0\right) \\
& =J^{-1} \circ Q_{D}\left(y_{n}^{0}, 0\right)=z_{n},
\end{aligned}
$$

so that $P \circ E=\operatorname{Id}_{Z}$.

\section{Further Results and Conjectures}

An obvious extension to Theorem 1 would be the following.

Conjecture. Suppose that $\left(Z_{0}, Z_{1}\right)$ is a Banach couple where $Z_{i}$ is a quotient of a subspace of $X_{i}$, for $i=0,1$, suppose that $X_{0}, X_{1}, V_{0}$ and $V_{1}$ are separable Banach spaces, and suppose that suitable dimension constraints are satisfied. Then there is a Banach couple $\left(A_{0}, A_{1}\right)$ such that

i) $A_{0}$ is isometric to $X_{0} \oplus V_{0}$, and $A_{1}$ is isometric to $X_{1} \oplus V_{1}$;

ii) for every exponential interpolation method $I$, the space $A_{I}$ contains a complemented subspace isomorphic to $Z_{I}$.

The second named author has a tentative proof of a local version of this result for the real interpolation method, which he hopes to publish later.

However, we can show the following.

Theorem 5. Suppose $\left(Y_{0}, Y_{1}\right)$ is a Banach couple, where $Y_{1} \subset Y_{0}$ with $\|y\|_{Y_{0}} \leq c\|y\|_{Y_{1}}$. Suppose further that $Y_{0}$ is a quotient space of a separable Banach space $X_{0}$, and $Y_{1}$ is a complemented subspace of a separable Banach space $X_{1}$, such that the codimensions of $Y_{0}$ in $X_{0}$ and $Y_{1}$ in $X_{1}$ are equal. Then there is a Banach couple $\left(A_{0}, A_{1}\right)$ such that

i) $A_{0}$ is isometric to $X_{0}$, and $A_{1}$ is isometric to $X_{1}$;

ii) given any exponential interpolation method of exponent $\theta$, there are maps $E: Y_{\min , \theta} \rightarrow$ $A_{I}$ and $P: A_{I} \rightarrow Y_{I}$, both of bounded norm, such that $P \circ E=\operatorname{Id}_{Y_{\min , \theta}}$

Corollary. There is a Banach couple $\left(A_{0}, A_{1}\right)$ such that $A_{0}$ is isometric to $l_{1}$, and $A_{1}$ is isometric to $l_{p}$, and for any exponential interpolation method $I$ of exponent $\theta$, the interme- 
diate space $A_{I}$ contains a subspace $V$ such that $l_{p / \theta, 1} \subseteq V \subseteq l_{p / \theta, \infty}$ with $c^{-1}\|x\|_{p / \theta, \infty} \leq$ $\|x\|_{V} \leq c\|x\|_{p / \theta, 1}$.

Corollary. There is a Banach couple $\left(A_{0}, A_{1}\right)$ such that $A_{0}$ has cotype 2 , and $A_{1}$ has cotype $p$ and is $K$-convex, and such that for all $0<\theta<1$, the real interpolation space $A_{\theta, 1}$ does not have cotype $r$ for any $r<p / \theta$.

This shows that a result of $\mathrm{Xu}[\mathbf{7}]$ cannot be improved. He showed that, if $\left(A_{0}, A_{1}\right)$ is a Banach couple such that $A_{1}$ is $K$-convex with cotype $p$, then for all $0<\theta<1$, the real interpolation space $A_{\theta, 1}$ has cotype $p / \theta$.

Proof of Theorem 5: Suppose that $Y_{0}$ is a quotient of $X_{0}$ by a subspace $Z_{0}$, and that $X_{1}=Y_{1} \oplus Z_{1}$. Denote the quotient map from $X_{0}$ to $Y_{0}$ by $Q$. Let $\left(z_{n}^{0} ; \tilde{\zeta}_{n}^{0}\right)$ be a total fundamental biorthogonal sequence for $Z_{0}$, and let $\left(z_{n}^{1} ; \tilde{\zeta}_{n}^{1}\right)$ be a total fundamental biorthogonal sequence for $Z_{1}$. Extend $\tilde{\zeta}_{n}^{0}$ to $\zeta_{n}^{0}$ on $X_{0}$ using the Hahn-Banach Theorem, and define $\zeta_{n}^{1}$ on $X_{1}$ by setting $\zeta_{n}^{1}(y, z)=\tilde{\zeta}_{n}^{1}(z)$. Without loss of generality, $\left\|\zeta_{n}^{0}\right\|=\left\|\zeta_{n}^{1}\right\|=1$.

To save chasing constants, let us assume that $I$ is exact. Let $\epsilon_{n}$ be a sequence of sufficiently small positive numbers, so that

$$
\delta=\sum_{n=1}^{\infty} \max \left\{\epsilon_{n}, \epsilon_{n}^{\theta}\right\} \max \left\{\left\|z_{n}^{0}\right\|,\left\|z_{n}^{1}\right\|\right\}<\infty,
$$

for all $0<\theta<1$. We will let $Y_{0} \oplus l_{\infty}$ be the ambient space. Let $M_{i}: X_{i} \rightarrow Y_{0} \oplus l_{\infty}$ be the mappings:

$$
\begin{aligned}
& M_{0}(x)=\left(Q(x),\left(\epsilon_{n} \zeta_{n}^{0}(x)\right)\right) \\
& M_{1}(x)=\left(Q(x),\left(\zeta_{n}^{1}(x)\right)\right) .
\end{aligned}
$$

Since $\left(z_{n}^{0} ; \tilde{\zeta}_{n}^{0}\right)$ and $\left(z_{n}^{1} ; \tilde{\zeta}_{n}^{1}\right)$ are both total biorthogonal systems, $M_{0}$ and $M_{1}$ are one to one. We let $A_{i}=M_{i}\left(X_{i}\right)$ with norms inherited from the domains of the functions.

Define $E: Y_{0} \rightarrow Y_{0} \oplus l_{\infty}$ by

$$
E(y)=(y, 0)
$$

We desire to show that $E\left(Y_{\min , \theta}\right) \subseteq A_{I}$, with $\|E\|_{Y_{\min , \theta} \rightarrow A_{I}}<\infty$. First, note that

$$
\left\|\left(0, \epsilon_{n} e_{n}\right)\right\|_{A_{0}}=\left\|z_{n}^{0}\right\|
$$

and

$$
\left\|\left(0, \epsilon_{n} e_{n}\right)\right\|_{A_{1}}=\epsilon_{n}\left\|z_{n}^{1}\right\|
$$


Hence,

$$
\left\|\left(0, \epsilon_{n}\right)\right\|_{A_{I}} \leq \epsilon_{n}^{\theta} \max \left\{\left\|z_{n}^{0}\right\|,\left\|z_{n}^{1}\right\|\right\}
$$

and so

$$
\left\|\left(0,\left(\epsilon_{n} \zeta_{n}^{0}(x)\right)\right)\right\|_{A_{I}} \leq \delta\|x\|_{X_{0}}
$$

Let $\nu>0$. For $y \in Y_{1}$, let $x \in X_{0}$ be such that $Q(x)=y$ with $\|x\|_{X_{0}} \leq(1+\nu)\|y\|_{Y_{0}}$. Then

$$
\begin{gathered}
\left\|\left(y,\left(\epsilon_{n} \zeta_{n}^{0}(x)\right)\right)\right\|_{A_{0}}=\|x\|_{X_{0}} \leq(1+\nu)\|y\|_{Y_{0}} \\
\|(y, 0)\|_{A_{1}}=\|y\|_{Y_{1}}
\end{gathered}
$$

and

$$
\begin{aligned}
\left\|\left(0,\left(\epsilon_{n} \zeta_{n}^{0}(x)\right)\right)\right\|_{A_{1}} & \leq \sum_{n} \epsilon_{n}\left\|z_{n}^{1}\right\|\|x\|_{X_{0}} \\
& \leq(1+\nu) \delta\|y\|_{Y_{0}} \leq(1+\nu) \delta\|y\|_{Y_{1}}
\end{aligned}
$$

So,

$$
\left\|\left(y,\left(\epsilon_{n} \zeta_{n}^{0}(x)\right)\right)\right\|_{A_{1}} \leq(1+\nu)(1+\delta)\|y\|_{Y_{1}}
$$

Therefore,

$$
\left\|\left(y,\left(\epsilon_{n} \zeta_{n}^{0}(x)\right)\right)\right\|_{A_{I}} \leq(1+\nu)(1+\delta)\|y\|_{Y_{0}}^{1-\theta}\|y\|_{Y_{1}}^{\theta} .
$$

Finally,

$$
\begin{aligned}
\|(y, 0)\|_{A_{I}} & \leq \delta\|x\|_{X_{0}}+(1+\nu)(1+\delta)\|y\|_{Y_{0}}^{1-\theta}\|y\|_{Y_{1}}^{\theta} \\
& \leq(1+\nu)(1+2 \delta)\|y\|_{Y_{0}}^{1-\theta}\|y\|_{Y_{1}}^{\theta} .
\end{aligned}
$$

This is sufficient to show that $\|E(y)\|_{A_{I}} \leq(1+\nu)(1+2 \delta)\|y\|_{Y_{\min , \theta}}$ as desired.

Now we let $P: Y_{0} \oplus l_{\infty} \rightarrow Y_{0}$ be the map

$$
P(y, a)=y
$$

Then $\|P\|_{A_{0} \rightarrow Y_{0}}=\|P\|_{A_{1} \rightarrow Y_{1}}=1$, and hence $\|P\|_{A_{I} \rightarrow Y_{I}} \leq 1$. Clearly $P \circ E=\operatorname{Id}_{Y_{\min , \theta}}$. 


\section{Acknowledgements}

We would like to express our warm thanks to G. Pisier for suggesting this line of research to us.

\section{References}

1 C. Bennett and R. Sharpley, Interpolation of Operators, Academic Press 1988.

2 J. Bergh and J. Löfström, Interpolation Spaces, Springer-Verlag 1976.

3 S.J. Dilworth, Complex convexity and the geometry of Banach spaces, Math. Proc. Camb. Phil. Soc. 99 (1986), 495-506.

4 J. Lindenstrauss and L. Tzafriri, Classical Banach Spaces I-Sequence Spaces, Springer-Verlag 1977.

5 J. Lindenstrauss and L. Tzafriri, Classical Banach Spaces II-Function Spaces, Springer-Verlag 1979.

6 A.I. Markushevich, On a basis in the wide sense for linear spaces, Dokl. Akad. Nauk 41 (1943), 241-244.

7 Q. Xu, Cotype of the spaces $\left(A_{0}, A_{1}\right)_{\theta 1}$, Geometric Aspects of Functional Analysis, Israel Seminar 1985-6, J. Lindenstrauss and V.D. Milman (Eds.), Springer Verlag 1987. 